\title{
Apego en niños con asistencia temprana a salas cuna en la ciudad de Punta Arenas (Chile): un estudio piloto
}

\author{
Rodrigo A. Cárcamo, Ph.D. \\ Profesor Titular Universidad de Magallanes, \\ Chile* \\ Sandy Lagos, Lic. \\ Licenciada Universidad de Magallanes, \\ Chile**
}

\author{
Alejandra García-Riquelme, Mg. \\ Profesora Asistente Universidad de \\ Magallanes, Chile ${ }^{\star \star \star}$ \\ Esteban Gómez, Ph.D. \\ Director Ejecutivo de Fundación América por

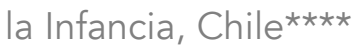

rodrigo.carcamo@umag.cl

\section{Resumen (analítico)}

Este estudio piloto tuvo por objetivo conocer los vínculos de apego en niños y niñas que asisten a salas cuna. Se aplicó la Escala de apego durante estrés (ADS) a 25 díadas madre-infante (cuyos niños y niñas tenían entre 10 y 14 meses de edad), que asistían a salas cuna en la ciudad de Punta Arenas. La muestra fue no probabilística intencional, mientras que el diseño no experimental, transversal, descriptivo. Los resultados indican que la distribución de los tipos de apego es similar al patrón universal; sin embargo, una prolongada estadía en salas cuna se asocia a un mayor riesgo de apego inseguro aun cuando los efectos de la edad de ingreso son controlados. Se discuten los alcances e implicancias para futuras investigaciones.

\section{Palabras-clave}

Centro de cuidado infantil, experiencia temprana, apego.

\section{Thesauro}

Tesauro de índice de términos de psicología
Para citar este artículo

Cárcamo, R. A., Lagos, S., García-Riquelme, A., \& Gómez, E. (2019). Apego en niños con asistencia temprana a salas cuna en la ciudad de Punta Arenas (Chile): un estudio piloto. Revista Latinoamericana de Ciencias Sociales, Niñez y Juventud, 17(2), 1-21. doi:10.11600/1692715x.17210

Historial

Recibido: 05.12.2017

Aceptado: 07.02.2018

Publicado: 30.06 .2019

Información artículo

El presente artículo se basa en la línea de investigación desarrollada por el primer autor y parte de la investigación realizada por la segunda autora (Apego en Niños con Asistencia Temprana a Salas Cuna en la Ciudad de Punta Arenas, Chile: Un Estudio Piloto), en la Universidad de Magallanes-Chile. Investigación iniciada en marzo del año 2009 y finalizada en marzo del 2010 (Fondecyt 11140663). Área: Psicología. 


\section{Attachment of children who experience early entry to daycare in Punta Arenas, Chile: a pilot study}

Abstract (analytical)

This pilot study had the objective of determining the distribution of attachment among children who experience early entry to daycare centers. The Attachment During Stress scale was implemented with 25 baby-mother dyads whose children were aged between 10 and 14 months old and were attending daycare centers in the city of Punta Arenas. The study used a deliberate non-probabilistic sample and it is a non-experimental, descriptive study. The results show that the distribution of attachment was similar to the universal pattern. However, a long period of time attending a daycare center is associated with a higher risk of insecure attachment even the effects of early entry are controlled. Implications and possibilities for future studies are also discussed.

Keywords

Child day care, early entry, attachment.

\section{O apego em crianças com atenção precoce em creches na cidade de Punta Arenas, Chile: um estudo piloto}

Resumo (analítico)

Este estudo piloto teve com objetivo conhecer os vínculos de apego em crianças que frequentam creches. Foi aplicada a Escala de Apego durante Stress (ADS) a 25 duplas materno - infante, cujas crianças estavam na faixa etária entre 10 a 14 meses de idade que frequentavam creches na cidade de Punta Arenas. A amostra foi não probabilística intencional e o desenvolvimento não experimental, transversal, descritivo. Os resultados indicam que a distribuição dos tipos de apego são similares ao padrão universal; contudo, a permanência prolongada em creches pode estar associada a um risco maior de apego inseguro mesmo quando os efeitos da idade de ingresso na creche são controlados. São discutidos os alcances e implicâncias para pesquisas futuras.

Palavras-chave

Creche infantil, experiência inicial, apego.

\section{Información autores}

[*] Psicólogo, Máster en Psicología Cognitiva (Universidad Autónoma de Madrid). Doctor en Psicopatología del Desarrollo desde una Perspectiva Educativa y Cultural (Universidad de Leiden, Holanda). Profesor Asociado, Departamento de Psicología, Universidad de Magallanes, Chile. Orcid: 0000-0002-9227-7132. Índice H5: 7. Correo electrónico: rodrigo.carcamo@umag.cl

[**] Psicóloga, Licenciada en Psicología, Universidad de Magallanes, Chile. Orcid: 0000-0003-4512-3058. Índice H5: 0 Correo electrónico: lagos.sandy@gmail.com

[***] Educadora de Párvulos, Licenciada en Educación, Máster en Estudios Latinoamericanos (Universidad de Leiden, Holanda). Profesora Instructora, Departamento de Educación, Carrera de Educación Parvularia, Universidad de Magallanes, Chile. Orcid: 0000-0003-3591-6185. Índice H5: 0 Correo electrónico:

alejandra.garcia@umag.cl

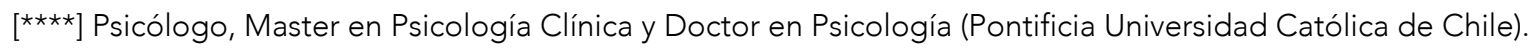
Director Ejecutivo de Fundación América por la Infancia. Orcid: 0000-0002-7642-1421. Índice H5: 11 Correo electrónico: esteban.gomez@americaporlainfancia.com 


\section{Introducción}

El cuidado no maternal profesional de niños y niñas a temprana edad es cada vez más común en muchas sociedades occidentales (De Grande, 2016), y en Chile, a partir de las políticas de ampliación de la cobertura de salas cuna, niños y niñas pequeños son atendidos en centros de cuidados diarios, con el fin de que sus madres puedan incorporarse al mundo laboral (Cárcamo, Van der Veer, Vermeer, \& Van IJzendoorn, 2014a; Noboa-Hidalgo \& Urzúa, 2012). En general, en el contexto del cuidado parental, son las mujeres quienes ejercen este rol de cuidado primario en sociedades como la chilena y otras similares de América Latina (Moreno-Roldán, Agudelo-Bedoya, \& Alzate-Pulgarín, 2018), situación que lleva a que cada vez sea mayor la cantidad de niños y niñas bajo el cuidado no maternal profesional. En Chile se produce un aumento sin precedentes de cobertura de la educación preescolar entre los años 2006 y 2009, incrementando el número de salas cuna públicas de 781 a 4281 (Junta Nacional de Jardines Infantiles, 2009). Luego de este periodo, el sistema preescolar ha ido creciendo de manera paulatina en alrededor de un $10 \%$ o menos anualmente (Ministerio de Educación-Mineduc, 2018). Dado el auge de la asistencia temprana a sala cuna, es importante conocer cuáles son sus efectos sobre diversas áreas del desarrollo infantil, como el desarrollo socioemocional, por ejemplo.

Son escasos los estudios fuera de Norteamérica y Europa sobre los efectos que este tipo de cuidados podría tener en los niños y niñas, ya que aun cuando hay pruebas de algunos beneficios en el ámbito cognitivo y en el desempeño escolar (Nichd Early Child Care Network, 2002), parece que estos beneficios están muy asociados a la calidad de los cuidados (Gerber, Whitebook, \& Weinstein, 2007; Huston, Bobbitt, \& Bentley, 2015). La evidencia más favorable a la fecha proviene de programas integrales de intervención infantil temprana, como el Early Head Start en Estados Unidos o el Sure Start en Inglaterra. Estos programas, enfocados en familias con acumulación de factores de riesgo psicosocial y que ofrecen prestaciones multimodales, han documentado efectos significativos 
a corto y mediano plazo en medidas de desarrollo cognitivo, psicomotor y socioemocional (Beeber et al., 2007).

Sin embargo, en dichos estudios se ha abordado principalmente el desarrollo cognitivo y psicomotor de niños y niñas en sistemas de cuidado diario infantil, pues hay menos evidencia en el área del desarrollo emocional, lo que resulta incluso más escaso en el contexto latinoamericano en general y en el chileno en particular.

\section{Antecedentes}

\section{Apego y desarrollo infantil temprano}

En la teoría del apego, desarrollada por Bowlby (a principios de la década de los cincuenta) se analizaban las posibles influencias adversas del cuidado maternal inadecuado durante la infancia y se enfatizaba en el desconsuelo que experimentan los niños y niñas separados de sus figuras de apego (Bowlby, 1952). Desde entonces, las relaciones que los niños y niñas desarrollan con sus cuidadores han sido un aspecto de gran relevancia en el ámbito multidisciplinario de las ciencias del desarrollo infantil temprano. Entre los trabajos fundacionales sobresale el de Ainsworth, Blehar, Waters y Wall (1978), quienes encontraron diferencias en la calidad de la interacción madre-hijo y su respectiva influencia sobre la formación del apego, llegando a clasificar patrones de apego que desplegaban los niños y niñas hacia su madre frente a una situación estresante. Estas observaciones pusieron en duda la mayoría de las teorías predominantes en los años cincuenta, las cuales explicaban la relación materno-infantil como secundaria y subordinada a una necesidad más primaria de alimentación; no obstante, los niños y niñas mostraban un intenso nivel de angustia cuando los separaban de sus padres (por ejemplo, en periodos de hospitalización), incluso cuando recibían cuidado físico adecuado (Van der Horst \& Van der Veer, 2009).

La formación del vínculo de apego es clave para el niño por lo significativa que resulta la figura de apego en cuanto a la disposición y accesibilidad que muestre, en particular cuando el niño se siente más desprotegido o asustado y necesita contención; de allí que esta relación se considerara una necesidad primaria. Para Bowlby (1952), el sistema vincular surge a partir de las ventajas de supervivencia que la proximidad y el contacto ofrecieron a los niños y niñas en el curso de la evolución humana.

Cuando el niño puede captar la disposición de su figura de apego ante sus demandas se siente más tranquilo y confiado para explorar, pues sabe que si necesita ayuda puede solicitarla y esta se le prestará (Bowlby, 1989). La proximidad física es uno de los factores 
considerados principales por Bowlby para la formación del vínculo de apego, debido a que las conductas de apego en relación con una figura determinada se desarrollan durante el primer año de edad. En los niños y niñas pequeños adquiere una mayor relevancia y notoriedad a causa de la constante búsqueda de protección y cercanía.

Respecto a la distribución de los estilos de apego en poblaciones normativas, en la revisión hecha por Mesman, Van IJzendoorn y Sagi-Schwartz (2016) se advierte que la distribución de los tipos de apego tiene algunas diferencias en los estudios analizados en varios países y dentro de los mismos países, particularmente en la proporción de apegos inseguros; sin embargo, el apego de tipo seguro, con excepción de un solo estudio, sería un referente de mayor prevalencia universal.

\section{Figuras múltiples de apego}

Actualmente, los niños y niñas pasan muchas horas — desde temprana edad- en salas cuna, por lo que surge la siguiente pregunta: ¿será posible que desarrollen vínculos de apego con el personal estable que los recibe, cuida y educa cada día? Bowlby dejó abierta esta posibilidad al plantear (contrario a lo que muchos profesionales siguen sosteniendo) que el apego se desarrolla con la madre o con quien ejerce el rol parental (Bowlby, 1989); es decir, el vínculo de apego o afectivo no se construiría únicamente con la madre, sino también con otras figuras significativas, por lo que se convertirían así en factores protectores (Moreno-Zavaleta \& Granada-Echeverri, 2014).

En algunas investigaciones (Ahnert, Pinquart, \& Lamb, 2006; Pallini, Baiocco, Baumgartner, Bellucci, \& Laghi, 2017) se han estudiado las relaciones de apego que los niños y niñas desarrollan con cuidadores alternativos, siendo la conclusión general que es posible construir esta clase de relaciones con otros cuidadores, con un patrón de apego seguro similar al universal, y que el tipo de apego que forman con el cuidador alternativo es independiente del que construyen con los padres. Esta posibilidad la han analizado Howes y Spieker (2016), quienes citan otros estudios en los que se aportan pruebas de la construcción de vínculos de apego entre los niños, niñas y educadoras en el contexto de centros de cuidado diario infantil; por ejemplo, ellos señalan que se veía una mayor presencia de apego seguro cuando había mayor cantidad de horas de contacto entre el niño o niña y la educadora, y que dirigían más conductas de apego hacia las educadoras que llevaban más tiempo en el centro, quienes a su vez eran más eficaces en regular el estrés de estos niños y niñas que aquellas que llevaban menos tiempo en el centro de cuidado (Howes \& Spieker, 2016). 
Factores asociados al apego en sistemas de cuidado infantil temprano

Aunque en la investigación se advierte que el cuidado y la crianza dados por los padres son un mecanismo de predicción más fuerte y consistente en el desarrollo de los niños y niñas que la experiencia temprana en jardines infantiles, esta igualmente tiene efectos identificables. En los últimos años se han abordado las consecuencias de la separación temprana de un niño y su figura de apego al ser inserto en una sala cuna, identificando algunas variables que hay que tomar en cuenta: edad de ingreso, tiempo semanal de asistencia, temperamento, características de los adultos responsables del cuidado y características del contexto familiar.

Sobre la edad de ingreso, Belsky (2006) señala en su revisión que los niños y niñas que asistían a sala cuna antes del año de edad tenían mayor probabilidad de desarrollar un apego inseguro a su figura parental, pero solo si esta poseía bajos niveles de sensibilidad parental. Para interpretar tales hallazgos, se propuso considerar que cuando el ingreso del niño a sala cuna ocurre más allá del año de edad, este tendría más recursos afectivos y cognitivos para comprender la experiencia, ya que además habría pasado mayor cantidad de tiempo en interacción cotidiana con su madre, lo que le daría mayor estabilidad tanto en la relación que construye con ella como en su disponibilidad.

Respecto al tiempo de asistencia, un estudio clásico que enciende las primeras alarmas sobre el riesgo de asistir a salas cuna por periodos prolongados es el de Belsky y Rovine (1988), quienes encontraron que más de 20 horas semanales de cuidados alternativos durante el primer año de vida constituían un factor de riesgo para el desarrollo de apego seguro a la madre. No obstante, esto no es concluyente, ya que en otros estudios se advierte que la exposición a cuidado no maternal a una edad más temprana y durante más horas predice en el niño conductas más agresivas y desobedientes, aunque también una mayor preparación para la actividad académica en el colegio (Belsky, Burchinal, McCarney, Vandell, Clarke-Stewart, \& Owen, 2007; Greenspan, 2003). Sin embargo, estos resultados son cuestionados en un metaanálisis por Roggman et al. (1994, en Santelices \& Olhaberry, 2009), y luego por Linting y Van IJzendoorn (2009), dejando abierto el debate sobre los efectos del tiempo de asistencia semanal a jardines infantiles en el desarrollo infantil, y motivando a incorporar más factores intervinientes en los análisis y nuevos estudios.

Un tercer factor que habría que considerar sería el temperamento del niño. Según Pluess y Belsky (2009), los efectos del cuidado no materno serían diferentes para cada niño, según el grado de susceptibilidad derivado de la negatividad emocional tempera- 
mental. Estos niños y niñas se verían más afectados por el cuidado no materno y presentarían mayor riesgo de verse afectados por cuidados de baja calidad. Sin embargo, en otros estudios se ha encontrado que cuando los cuidadores de jardines infantiles muestran actitudes positivas en forma frecuente, los niños y niñas desarrollan una vinculación segura hacia ellos, con independencia del temperamento del niño, el que no aparece como predictor ni moderador del tipo de vínculo construido (De Schipper, Tavecchio, \& Van IJzendoorn, 2008).

El cuarto grupo de factores identificados en la bibliografía existente hace referencia a las características del personal de centros de cuidado diario. En este ámbito, la evidencia sugiere que mientras más grande es el grupo y menos entrenado el personal, más caos se genera en el lugar y menos sutil se vuelve la interacción entre los niños y niñas y el personal, interfiriendo negativamente en las funciones de empatía, cuidados sensibles y regulación psicobiológica de la experiencia del infante (Greenspan, 2003). En un estudio conducido por Gerber et al. (2007), se identificaron como factores predictores de mayor sensibilidad del cuidador el estatus de acreditación del centro, un menor tamaño de este y la calidad de su infraestructura. Un hallazgo relevante de dicho estudio fue que el entrenamiento que recibía el cuidador también era un predictor de mayor sensibilidad, que incluso moderaba los efectos negativos de la depresión del cuidador.

Por último, el factor del contexto familiar que aparece como más relevante a la hora de moderar las conductas y respuestas de los niños y niñas al ingresar a jardines infantiles es la sensibilidad parental. La sensibilidad materna o paterna ha mostrado ser una base para la implementación de estrategias de disciplina positiva, donde además es clave la alianza con el padre, quien coopera en el proceso (Alink et al., 2009). El papel de la madre se ha valorado también en otros estudios en los que se ha descubierto que el mal manejo que esta tenga del estrés familiar se relaciona con los comportamientos problema y con la dificultad de adaptación del niño al contexto de jardines infantiles (De Schipper, Van IJzendoorn, \& Tavecchio, 2004).

\section{El contexto chileno}

Una publicación relevante para el objetivo de esta investigación es la de Olhaberry (2011), quien abordó el estudio de la calidad de las interacciones madre-hijo en 80 díadas, con niños y niñas entre 4 y 15 meses, usando el instrumento Care-Index (Crittenden, 2005) para comparar dos grupos, según asistencia o no a salas cuna. Se encontró que las díadas sin asistencia tuvieron interacciones más afectuosas, con un tamaño de efecto 
mediano, y que aquellas díadas con edades de ingreso inferiores a seis meses presentaron interacciones de peor calidad, con tamaños de efectos medianos y grandes. En este estudio se cuestionan los efectos de la asistencia a salas cuna a temprana edad, específicamente antes de los seis meses, sobre el desarrollo de la interacción madre-hijo/a.

En otro estudio en esta línea, realizado en Chile por Muñoz y Santelices (en prensa), se evaluó la calidad de la interacción de las educadoras en una muestra de 124 díadas con niños y niñas que asisten a salas cuna de la Junji, a las cuales se les aplicó también el Care-Index, y se reportó una serie de factores asociados. Se halló que una mayor cantidad de años de trabajo de la educadora en la sala cuna actual se relacionó con un mayor nivel de sensibilidad afectiva, pero no cognitiva; una formación superior completa se asoció a niveles más altos de sensibilidad general y cognitiva, pero no afectiva; cabe señalar que las educadoras de párvulos mostraron, en general, mayor sensibilidad en todas las áreas estudiadas que las técnicas en párvulos. Estos hallazgos se orientan a reafirmar que los años de entrenamiento y formación se asocian a mayores niveles de sensibilidad en la interacción, tal como se señala en la bibliografía especializada.

En razón de la importancia que han demostrado tener los procesos de conformación de un apego seguro en el desarrollo humano, este surge como un área relevante de investigación.

Más allá de la valiosa contribución de estos estudios realizados en el país, sigue siendo necesario desarrollar investigación específica sobre estilos de apego en contextos de salas cuna y jardines infantiles, así como también sobre los factores que influyen en su conformación. La idea con este estudio es contribuir al avance en el tema, en el ámbito exploratorio, y con una muestra del extremo sur del país.

La hipótesis del estudio fue, en primer lugar, que la muestra tendría una mayor representación de niños y niñas con apegos inseguros, con respecto a la distribución en poblaciones normales de Chile y en otros países, debido a su inserción temprana en cuidados no parentales durante extensas jornadas; en segundo término, se pretendió evaluar si algunas de las variables demográficas tenían relación con el tipo de apego mostrado por las díadas; y, en tercera instancia, si la inserción temprana y la estadía prolongada en salas cuna podrían tener efecto sobre el tipo de apego desarrollado por las díadas. 


\section{Método}

\section{Participantes}

La muestra se obtuvo por medio de un muestreo no probabilístico intencional, en el que se contactó a varias madres a través de salas cuna públicas Junji (Junta Nacional de Jardines Infantiles) en la ciudad de Punta Arenas. Participaron 25 díadas madre-infante ( $52 \%$ niñas), con edades que fluctuaban entre los 10 y 14 meses $(M=12.32, D S=1.49)$, que asistían a salas cuna por igual cantidad de horas semanales (36.5) y cuyas edades de ingreso variaban entre los 3 y 8 meses de edad. Las madres, por su parte, tenían una edad promedio de 26.4 años $(D S=6.65)$ y un $60 \%$ de ellas con estudios secundarios completos (tabla 1). Como criterio de exclusión se determinó no incluir en el estudio a madres que presentaran algún tipo de psicopatología o que se encontraran en una situación de alta vulnerabilidad o riesgo.

\section{Instrumento}

Para clasificar a cada díada en una categoría de apego se empleó la Escala de apego durante estrés (ADS) (Massie \& Campbell, 1983). Esta entrega indicadores respecto de la calidad del apego por medio de la observación de la interacción entre el infante y su cuidador significativo - generalmente la madre-durante situaciones de estrés leve o moderado, que pueden ser el control pediátrico, experiencias de separación como en el procedimiento de una situación extraña o dejar al niño en la sala cuna (jardines infantiles), al igual que episodios de interacción en el hogar (Massie \& Campbell, 1983). Para este estudio se utilizaron grabaciones de interacciones en un contexto cotidiano para el niño, que incluyeron muda, alimentación y juego libre en el hogar. La escala cuenta con evidencia de su validez psicométrica (Cárcamo, Van IJzendoorn, Vermeer, \& Van der Veer, 2014b; Nóblega et al., 2019). 


\section{Tabla 1}

Características de la muestra

\begin{tabular}{|c|c|c|c|c|c|c|}
\hline Características de los niños y niñas & M & DS & MIN & MAX & $\mathbf{N}$ & $\%$ \\
\hline Edad niños (en meses) & 12.3 & 1.49 & 10 & 14 & & \\
\hline \multicolumn{7}{|l|}{ Sexo } \\
\hline Niño & & & & & 12 & 48 \\
\hline Niña & & & & & 13 & 52 \\
\hline \multicolumn{7}{|l|}{ Orden de nacimiento } \\
\hline Primero & & & & & 13 & 52 \\
\hline Segundo & & & & & 6 & 24 \\
\hline Tercero & & & & & 6 & 24 \\
\hline Edad de ingreso a sala cuna & 5.0 & 1.11 & 3 & 8 & & \\
\hline 3 meses & & & & & 1 & 4 \\
\hline 4 meses & & & & & 9 & 36 \\
\hline 5 meses & & & & & 6 & 24 \\
\hline 6 meses & & & & & 8 & 32 \\
\hline 8 meses & & & & & 1 & 4 \\
\hline Meses de permanencia en sala cuna & 7.36 & 1.38 & 6 & 10 & & \\
\hline Características de la madre & M & DS & MIN & MAX & $\mathbf{N}$ & $\%$ \\
\hline Edad madres en años & 26.4 & 6.65 & 17 & 41 & & \\
\hline Número de hijos de la madre & 1.72 & 0.84 & 1 & 3 & & \\
\hline Número de habitantes en el hogar & 4.28 & 0.94 & 2 & 6 & & \\
\hline Escolaridad madres (en años) & 9.54 & 3.22 & & & & \\
\hline Básica & & & & & 1 & 4 \\
\hline Media incompleta & & & & & 4 & 16 \\
\hline Media completa & & & & & 15 & 60 \\
\hline Estudios técnicos & & & & & 4 & 16 \\
\hline Estudios universitarios & & & & & 1 & 4 \\
\hline \multicolumn{7}{|l|}{ Estado civil madres } \\
\hline Casadas & & & & & 13 & 52 \\
\hline Convivencia & & & & & 3 & 12 \\
\hline Soltera & & & & & 9 & 36 \\
\hline
\end{tabular}

La escala posee dos pautas de observación — una para la madre (o cuidador) y otra para el niño-, cada una de las cuales tiene siete indicadores de conductas de apego: mirada, vocalización, sostén, contacto físico hacia el otro, contacto físico de rechazo, afecto 
y proximidad. En cada indicador se asigna un puntaje de 1 a 5, dependiendo del tipo de la frecuencia e intensidad del comportamiento, o se consigna como conducta no observada. En la escala ADS no se usa un puntaje numérico ni se emplean promedios para definir una categoría determinada, pero las díadas se clasificaron, en un primer momento, en función de la frecuencia de puntajes observados en cada conducta de apego; para esto, los puntajes 1 y 2 corresponden a conductas de apego inseguro-evitativas, los puntajes 3 y 4 a conductas de apego seguras y los puntajes 5 a conductas de apego inseguro-resistente; no obstante, en un estudio de validación del instrumento (Cárcamo et al., 2014b) se advierte que el instrumento tiene dificultades para captar apego de tipo resistente, por lo que solo se utilizaron las categorías seguro y no seguro.

Respecto a sus propiedades psicométricas, ha mostrado indicadores de consistencia interna medida con alfa de Cronbach de 0.737 al ingreso y 0.736 al egreso para la pauta de infantes y 0.839 al ingreso y 0.824 al egreso para la pauta de la madre; una confiabilidad interevaluador de 0.83 a 0.89 (Massie \& Campbell, 1983) y su validez convergente se ha determinado usando la «medida estándar de oro» para evaluar apego (Ainsworth et al., 1978), arrojando resultados adecuados para distinguir entre apego seguro y no seguro $\left(\mathrm{x}^{2}=13.20, d=0.96\right)$ (Cárcamo et al., 2014b).

\section{Procedimiento}

Inicialmente, se hizo el contacto con los miembros de la Junta Nacional de Jardines Infantiles (Junji) de Punta Arenas, a quienes se les explicó la investigación e invitó a participar, lo que permitió el contacto con las familias de niños y niñas que estaban asistiendo a las salas cuna de su administración. Se procedió al contacto con las directoras y con las educadoras del nivel sala cuna para comunicar los objetivos y metodología del estudio y, a la vez, coordinar la entrega de una circular confeccionada para los padres, en la que se explicó el estudio, así como su objetivo y requerimientos. La circular venía con una colilla que se debía devolver al jardín si la madre aceptaba participar, para que la contactaran telefónicamente con el fin de concertar una cita en el hogar de la familia para filmar la escala ADS. Se hizo entrega del consentimiento informado al adulto responsable y se completó una anamnesis breve. Luego se procedió a la filmación (realizada por tercer autor), en la que se pidió a la madre adoptar las conductas de alimentación, muda y juego con el niño/a que normalmente ocurren en casa, sin entregar otra instrucción.

La codificación de los videos estuvo a cargo de dos observadores entrenados (primer y segundo autor), quienes no disponían de información de las díadas: la confiabilidad 
interobservadores fue kappa $=0.70$ para el total de la muestra $(n=25)$. Además, se evaluó la consistencia interna con alfa de Cronbach para cada codificador, obteniendo $0.753 \mathrm{y}$ o.840 para la pauta del infante, y para la pauta de la madre 0.936 y 0.945 , respectivamente.

\section{Análisis de datos}

Para el análisis de los datos sobre distribución de tipos de apego en la muestra se ocuparon estadísticos descriptivos, en tanto que para estudiar la relación entre las variables sociodemográficas consideradas en el estudio y el tipo de apego se condujeron análisis bivariados, usando la prueba chi cuadrado o $t$ de Student, según correspondiera. Finalmente, se utilizó la prueba $t$ de Student y se hizo una regresión logística para analizar la influencia del tiempo de estadía y edad de ingreso a sala cuna, sobre el tipo de apego identificado. Los análisis se efectuaron usando el programa SPSS ${ }^{\mathrm{TM}}$.

\section{Resultados}

En relación con el primer interrogante, se ha analizado cómo se distribuyen los tipos de apego en la muestra. Tomando en cuenta que el instrumento discrimina más adecuadamente entre apego seguro e inseguro, se utilizaron ambas clasificaciones.

Se observaron un $68 \%$ de apego seguro y un $32 \%$ de apego inseguro, lo cual se asemeja bastante a la distribución en poblaciones normativas chilenas (Lecannelier, Kimelman, González, Núñez, \& Hoffmann, 2008) y a la escala mundial (Mesman et al., 2016), pero no se confirma una sobrerrepresentación de apegos inseguros, como se esperaba. Sin embargo, los estudios que se mencionan de poblaciones normativas usan un instrumento diferente, la medida de oro, o gold standard, del apego (procedimiento de la situación extraña).

Por otro lado, se analizaron diferencias según el tipo de apego en las variables demográficas involucradas en este estudio: edad de la madre $(t(23)=-1.391, p=0.177)$, nivel educativo de la madre $\left.x^{2}(4, N=25)=5.08, p=0.279\right)$, número de hijos $(t(23)=-1.435$, $p=0.165)$, habitantes en el hogar $(t(23)=1.281, p=0.213)$, edad del niño $(t(23)=1.956$, $p=0.063)$ y orden de nacimiento del niño $\left.x^{2}(2, N=25)=2.49, p=0.287\right)$, sin encontrar diferencias significativas.

Especial mención merece el estado civil de la madre, ya que cuando se analizó el tipo de apego en función de la relación con el padre del niño (soltera, casada o en conviven- 
cia) no hubo relación significativa, pero cuando se agrupó a las madres de acuerdo con dos grupos (por una parte soltera y por otra en convivencia o casada), los resultados mostraron una relación marginalmente significativa $\left.\mathrm{X}^{2}(1, N=25)=4.28, p=0.11\right)$, con mayor prevalencia de apego seguro en el grupo de casadas o en convivencia ( $81 \%)$ versus solteras $(44 \%)$.

Al comparar los dos grupos de apego (inseguro y seguro) según el tiempo que llevaban asistiendo a la sala cuna, se encontró que los infantes con apego seguro $(M=6.66$, $D S=0.80$ ) llevaban menos meses de permanencia en sala cuna que los infantes con apego inseguro $(M=9.00, D S=0.76)$, diferencia estadísticamente significativa $(t(23)=7.18$, $p<0.001)$, y con un tamaño de efecto grande $(d=3.14)$. Al mirar los datos descriptivos, se observa que los niños y niñas que han estado seis o siete meses en sala cuna presentan solo apego seguro, mientras que los niños y niñas que han estado nueve o diez meses muestran únicamente apego inseguro (tabla 2).

\section{Tabla 2}

Frecuencias de clasificación de apego y meses en sala cuna

\begin{tabular}{|c|c|c|}
\hline \multirow{2}{*}{$\begin{array}{l}\text { Meses en } \\
\text { sala cuna }\end{array}$} & \multicolumn{2}{|c|}{ Apego } \\
\hline & Inseguro & Seguro \\
\hline 6 & 0 & 10 \\
\hline 7 & 0 & 4 \\
\hline 8 & 2 & 3 \\
\hline 9 & 4 & 0 \\
\hline 10 & 2 & 0 \\
\hline
\end{tabular}

Al estudiar la variable edad de ingreso, también se observaron diferencias en los promedios para el grupo de infantes con apego seguro $(M=5.41, D S=1.06)$, frente al grupo con apego inseguro $(M=4.12, D S=0.64)$; así, hubo significancia estadística $(t(23)=-3.14, p=0.005)$ y tamaño de efecto grande $(d=1.47)$.

Ambas variables, meses en sala cuna y edad de ingreso, se correlacionaron en forma significativa con el tipo de apego (tabla 3 ). 


\section{Tabla 3}

Coeficientes de correlación de Pearson para tipo de apego y variables

\begin{tabular}{lc}
\hline & Tipo de apego \\
\hline Edad de ingreso a sala cuna & $0.548^{\star \star}$ \\
Meses en sala cuna & $-0.832^{\star \star}$ \\
Residual no estandarizado & $-0.700^{\star \star}$ \\
\hline
\end{tabular}

Nota. $* * \mathrm{p}<\mathrm{O}, \mathrm{O} 1$.

Estos hallazgos muestran que los niños y niñas con apego inseguro han ingresado a la sala cuna a más temprana edad y han asistido a ella por más tiempo que el grupo de apego seguro. Al controlar por la edad de ingreso, se pudo advertir que el tiempo de permanencia en sala cuna continuó estando fuertemente asociado con el apego seguro ( $p=0.011)$. Cuando el número de meses en sala cuna (controlado por la edad de ingreso) se incrementa en una unidad, la razón de probabilidad (odds ratio) de tener un apego seguro disminuye con un factor de 0.135 (tabla 4 ).

\section{Tabla 4}

Resultados de la regresión logística para tipo de apego (método Enter)

\begin{tabular}{cccccccccc}
\hline & & & & & & \multicolumn{3}{c}{$95 \%$ I.C. para Exp(B) } \\
& & B & S.E. & Wald & gl & Sig. & Exp(B) & Bajo & Alto \\
\hline \multirow{2}{*}{ Paso 1} & Meses en sala cuna & -2.005 & 0,788 & 6.476 & 1 & 0.011 & 0.135 & 0.029 & 0.631 \\
& constante & 1.627 & 0,838 & 3.770 & 1 & 0.052 & 5.089 & & \\
\hline
\end{tabular}

\section{Discusión}

Los resultados obtenidos en este estudio piloto no permiten confirmar la hipótesis planteada inicialmente acerca de una mayor prevalencia de tipo inseguro de apego en niños y niñas asistentes a sala cuna, en relación con la población normativa. Sin embargo, en un análisis más detallado se encontró que cuando la edad de ingreso a sala cuna es posterior a los cinco o seis meses de edad, existe mayor tendencia hacia el tipo de apego seguro. Esto parece indicar que es más favorable para el desarrollo de un apego seguro pasar un tiempo importante durante los primeros meses de vida bajo el cuidado familiar 
y no grupal exclusivo y, de ser necesarios los cuidados no parentales, estos debieran postergarse a una edad mayor.

A la luz de la teoría, esto podría deberse a que durante el tiempo de cuidados parentales el infante tendría una mayor probabilidad de desarrollar conductas de apego seguro en conjunto con su cuidador principal, lo que le permitiría asimilar de mejor manera la experiencia de estrés y separación. Además, se encontró evidencia de que cuando es mayor el tiempo de permanencia en sala cuna, aumenta considerablemente la posibilidad de encontrar díadas con tipos de apego inseguro. Se aprecia que los niños y niñas con menos meses de permanencia en sala cuna presentan apego seguro, mientras que quienes tienen mayor tiempo de permanencia en sala cuna manifiestan una proporción mayor de apego inseguro.

Cabe anotar que el tiempo de permanencia no se refiere necesariamente a un ingreso temprano, sino tan solo a permanecer mayor número de meses bajo cuidados no parentales. Lo anterior confirma la importancia de promover el cuidado familiar más allá de los cuatro meses, respaldando la reforma realizada en el país a partir de octubre del 2011 mediante la cual se modifica la Ley 20545, que introduce reformas e incorpora nuevas normativas al Código del Trabajo, en la que se destaca el incremento del descanso posnatal de 12 semanas hasta un total de 24 semanas, con la posibilidad de traspasar parte de ese posnatal al padre.

Por otro lado, el análisis de variables sociodemográficas presentó datos interesantes y de acuerdo con la evidencia teórica hallada. Entre ellos se encontró que la distribución del tipo de apego se vería afectada por el estado civil de la madre y que sería mayor hacia el tipo de apego seguro cuando la madre está viviendo en pareja. Esto se explica en diversos estudios (Candelaria, Teti, \& Black, 2011), desde la idea de que la madre, cuando está casada o convive con una pareja y tiene una relación de pareja satisfactoria o con un mayor nivel de compromiso formal, puede recibir más apoyo social (emocional, económico, instrumental) de su pareja, lo que facilita su proceso de construcción de un apego seguro. Sin embargo, en este punto cabe mencionar una nota precautoria, ya que tales hallazgos no implican que se deba seguir naturalizando la distribución tradicional de roles de género en la crianza; ello especialmente a la luz de las nuevas tendencias que proponen la incorporación activa de los padres o figuras parentales masculinas en forma directa - y no únicamente como soporte de la madre - en la crianza. Así las cosas, en futuros estudios se debería evaluar el apego del infante a la madre, el padre u otra figura 
que cumpla un papel parental significativo. Con respecto al sexo del infante, no parece haber diferencias significativas que influyan en el desarrollo del tipo de apego.

En cuanto a la edad de la madre, se encontró que el tipo de apego seguro se halla en mayor proporción cuando la madre supera los 22 años, ya que entre los 17 y 21 años se advirtió una marcada tendencia hacia el tipo de apego inseguro. La mayor edad de la madre se ha considerado en otros estudios como un factor protector (Bakermans-Kranenburg, Van IJzendoorn, \& Kroonenberg, 2004), asociado positivamente a un tipo de apego seguro.

\section{Conclusiones}

Si bien los efectos de la asistencia a salas cuna es un tema que aún se encuentra en discusión, en la bibliografía reciente se sugiere que sus efectos dependen de múltiples factores, tales como el sexo de los niños, edad, nivel socioeconómico y etnicidad (Busse \& Gathmann, 2018; Cárcamo, Vermeer, Van der Veer, \& Van IJzendoorn, 2016). Busse y Gathmann (2018) reportan que las niñas se benefician más que los niños de la atención temprana a jardines infantiles, en resultados cognitivos y no cognitivos, mientras que en relación con efectos de tipo socioafectivo, por ejemplo, atenuar el impacto de una madre autoritaria con comportamiento físicamente agresivo solo beneficia a los niños (Muñoz et al., 2017).

En cuanto a vulnerabilidad étnica y socioeconómica, los efectos de la atención temprana aparecen como positivos en los grupos más vulnerables, sobre todo en las conductas de vinculación afectiva con las madres (Cárcamo et al., 2016). A la luz de estos trabajos, los resultados del presente estudio aportan a la discusión sobre la edad de inicio a la atención de tiempo completo en salas cuna o jardines infantiles, sugiriendo que en el primer año de vida el tiempo de cuidado no parental podría ser un factor de riesgo para el desarrollo del vínculo de apego.

Aun valorando los avances recientes que ha mostrado Chile en esta materia, resulta preocupante que quienes ingresan mayoritariamente en forma temprana a salas cuna de la Junji sean aquellos niños y niñas de padres que tienen mayor situación de vulnerabilidad y menos posibilidades de brindar cuidados parentales; lo anterior, por el hecho de ser, por ejemplo, madres trabajadoras (muchas con trabajos precarios, temporales, independientes o por honorarios) y con todas las condiciones de riesgo que el entorno social puede presentar para ellos y su relación vincular. 
En lo referente al posible deterioro del vínculo de apego, sería necesario hacer un estudio longitudinal con muestras más representativas. En cuanto a la idea relacionada con la temprana inserción y la tendencia hacia un tipo de apego seguro, sería importante realizar un estudio con un grupo que asista a sala cuna en una proporción menor de horas de cuidados no parentales a la semana y un grupo con cuidados parentales exclusivos, utilizando medidas adecuadas de sensibilidad que parecen ser un importante predictor del bienestar infantil y del desarrollo de apego seguro, que permitan dirigir adecuadas intervenciones tempranas (Salinas-Quiroz \& Posada, 2015).

Hay que considerar las limitaciones de los resultados obtenidos, dadas ciertas características de este estudio, tales como la falta de un grupo control que permitiera hacer, por ejemplo, análisis comparativos; el tamaño reducido de la muestra, aun cuando representó el $45 \%$ de la población que cumplía los requisitos para participar en el estudio, y evaluar aspectos de la calidad de las salas cuna, tanto en su estructura como en sus procesos de cuidados.

Este estudio se ha planteado como un estudio piloto, el cual se pretende reproducir en una muestra mayor, con grupo de control y con mayor heterogeneidad en cuanto al tipo y cantidad de cuidados recibidos, en la que se considere también la calidad de estos, para así comprender de manera más profunda la interacción de otras variables intervinientes en el desarrollo del vínculo de apego en los niños y niñas que reciben cuidados no parentales y evaluar sus beneficios y riesgos. Algunos aspectos que hay que tomar en cuenta son la evaluación de la calidad del ambiente familiar, la sensibilidad parental, la estructura familiar y el nivel de vulnerabilidad, la calidad de las salas cuna y la sensibilidad de los cuidadores con instrumentos adaptados a nuestro contexto (Santelices, Carvacho, Farkas, León, Galeguillos, \& Himmel, 2012); así como también el tipo de formación de las educadoras y técnicos de la educación preescolar, ya que hay evidencia de que en nuestro contexto parece que las representaciones e información general que las cuidadoras profesionales tienen sobre la teoría del apego no son del todo apropiadas (Huaiquián-Billeke, Mansilla-Sepúlveda, \& Lasalle-Rivas, 2016).

Gracias a este estudio piloto se pudieron conocer algunos resultados que podrían guiar futuras investigaciones en el área y se reveló una importante discusión sobre los beneficios o perjuicios de los cuidados no parentales a temprana edad, sobre todo la edad de ingreso a salas cuna, considerando además los resultados similares a los que llega Olhaberry (2011) en el estudio también realizado con muestras chilenas.

Así las cosas, parece imperioso generar más investigación en Chile sobre este tema y con mayor sofisticación del diseño, que nos permita responder a toda la complejidad que este problema presenta. 


\section{Referencias}

Ahnert, L., Piquart, M., \& Lamb, M. E. (2006). Security of children's relationships with non-parental care providers: a meta-analysis. Child Development, 74, 664-679. https://doi.org/10.1111/j.1467-8624.2006.00896.x

Ainsworth, M. D. S., Blehar, M. C., Waters, E., \& Wall, S. (1978). Patterns of attachment: A psychological study of the strange situation. Hillsdale: Erlbaum.

Alink, L. R. A., Mesman, J., Van Zeijl, J., Stolk, M. N., Juffer, F., Koot, H. M. ... van IJzendoorn, M. (2009). Maternal sensitivity moderates the relation between negative discipline and aggression in early childhood. Social Development, 18, 99-120. https://doi.org/10.1111/j.1467-9507.2008.00478.x

Bakermans-Kranenburg, M., Van IJzendoorn, M., \& Kroonenberg, P. (2004). Differences in attachment security between African-American and white children: ethnicity or socio-economic status? Infant Behavior \& Development, 27, 417-433. https://doi.org/ 10.1016/j.infbeh.2004.11.002

Beeber, L. S., Chazan-Cohen, R., Squires, J., Harden, B. J., Boris, N. W., Heller, S. S., \& Malik, N. M. (2007). The early promotion and intervention research consortium (EPirc): Five approaches to improving infant/toddler mental health in Early Head Start. Infant Mental Health Journal, 28, 130-15o. https://doi.org/10.1002/imhj.20126 Belsky, J. (2006). Early child care and early child development: Major findings of the NICHD study of early child care. European Journal of Developmental Psychology, 3(1), 95-110. https://doi.org/10.1080/17405620600557755

Belsky, J., \& Rovine, M. J. (1988). Non-maternal care in the first year of life and the security of infant-parent attachment. Child Development, 59, 157-167. https://doi.org/ 10.1111/j.1467-8624.1988.tbo3203.x

Belsky, J., Burchinal, M., McCarney, K., Vandell, D., Clarke-Stewart, K., \& Owen, M. (2007). Are there long-term effects of early child care? Child Development, 78, 681-701. https://doi.org/10.1111/j.1467-8624.2007.01021.x

Bowlby, J. (1952). Maternal care and mental health. Ginebra: World Health Organization. Bowlby, J. (1989). Una base segura: aplicaciones clínicas de una teoría del apego. Barcelona: Paidós.

Busse, A., \& Gathmann, C. (2018). Free daycare and its effects on children and their families. Bonn: Institute of Labor Economics (IZA). 
Candelaria, M., Teti, D. M., \& Black, M. M. (2011). Multi-risk infants: Predicting attachment security from sociodemographic, psychosocial, and health risk among African-American preterm infants. Journal of Child Psychology and Psychiatry, 52, 870877. https://doi.org/10.1111/j.1469-7610.2011.02361.x

Cárcamo, R. A., van der Veer, R., Vermeer, H. J., \& Van IJzendoorn, M. H. (2014a). From foundling homes to day care: A historical review of childcare in Chile. Cadernos de Saúde Pública, 31(3), 461-471. http://dx.doi.org/10.1590/0102-311X00060613

Cárcamo, R. A., Van IJzendoorn, M. H., Vermeer, H. J., \& van der Veer, R. (2014b). The validity of the Massie-Campbell Attachment during Stress Scale (ADS). Journal of Child and Family Studies, 23, 767-775. https://doi.org/10.1007/s10826-013-9728-Z

Cárcamo, R. A., Vermeer, H. J., van der Veer, R., \& Van IJzendoorn, M. H. (2016). Early full-time daycare, mother-child attachment and quality of the home environment in Chile: Preliminary findings. Early Education and Development, 27, 457-477. https://doi.org/10.1080/10409289.2016.1091971

Crittenden, P. (2005). Care-Index para toddlers: manual de codificación. Miami: Family Relations Institute.

De Grande, P. (2016). Diseñado para bebés: objetos y prácticas en el primer año de vida. Revista Latinoamericana de Ciencias Sociales, Niñez y Juventud, 14(1), 287-30o. https://doi.org/10.1160o/1692715x.1417090915

De Schipper, J., van IJzendoorn, M. H., \& Tavecchio, L. (2004). Stability in center day care: Relations with children's well-being and problem behavior in day care. Social Development, 13, 531-550. https://doi.org/10.1111/j.1467-9507.2004.00282.x

De Schipper, J., Tavecchio, L., \& van IJzendooorn, M. H. (2008). Children's attachment relationships with day caregivers: Associations with positive caregiving and the child's temperament. Social Development, 7, 454-47o. https://doi.org/10.1111/j. 1467-9507.2004.00282.x

Gerber, E., Whitebook, M., \& Weinstein, R. (2007). At the heart of child care: Predictors of teacher sensitivity in center-based child care. Early Childhood Research Quarterly, 22, 327-346. https://doi.org/10.1016/j.ecresq.2006.12.003

Greenspan, S. (2003). Child-care research: A clinical perspective. Child Development, 74, 1064-1068. https://doi.org/10.1111/1467-8624.00591

Howes, C., \& Spieker, S. (2016). Attachment relationships in the context of multiple caregivers. En J. Cassidy, \& P. R. Shaver (Eds.), Handbook of attachment: Theory, research, and clinical applications (pp. 314-329). Nueva York: Guilford. 
Huaiquián-Billeke, C., Mansilla-Sepúlveda, J., \& Lasalle-Rivas, V. (2016). Apego: representaciones de educadoras de párvulos en jardines infantiles en Temuco, Chile. Revista Latinoamericana de Ciencias Sociales, Niñez y Juventud, 14(2), 1119-1129.

https://doi.org/10.1160o/1692715x.14216100115

Huston, A. C., Bobbitt, K. C., \& Bentley, A. (2015). Time spent in child care: How and why does it affect social development? Developmental Psychology, 51(5), 621-634. https://doi.org/10.1037/aoo38951

Junta Nacional de Jardines Infantiles. (2009). Cuenta pública 2006-2009.

Lecannelier, F., Kimelman, M., González, L., Núñez, C., \& Hoffmann, M. (2008). Evaluación de patrones de apego en infantes durante su segundo año en dos centros de atención de Santiago de Chile. Revista Argentina de Clínica Psicológica, 17, 197-207.

Linting, M., \& van IJzendoorn, M. H. (2009). Dissipating or diffusing aggression after non-maternal childcare? Commentary on a novel hypothesis. Social Development, 18, 239-246. https://doi.org/10.1111/j.1467-9507.2008.00512.x

Massie, H., \& Campbell, B. K. (1983). The Massie-Campbell scale of mother-infant attachment indicators during stress. En J. Call, E. Galenson, \& R. Tyson (Eds.), Frontiers of infant psychiatry (pp. 394-412). Nueva York: Basic Books.

Mesman, J., van IJzendoorn, M. H., \& Sagi-Schwartz, A. (2016). Cross-cultural patterns of attachment: Universal and contextual dimensions. En J. Cassidy \& P. R. Shaver (Eds.), Handbook of attachment: Theory, research, and clinical applications (pp. 852-877). Nueva York: Guilford Press.

Ministerio de Educación. (2018). Más salas cuna y jardines infantiles para Chile: Programa de aumento de cobertura 2014-2018. Santiago de Chile: Autor. Recuperado de: https://parvularia.mineduc.cl/cobertura/

Moreno-Roldán, M. R., Agudelo-Bedoya, M. E., \& Alzate-Pulgarín, V. (2018). Voces a escuchar en el cuidado: ¿qué dicen los niños y las niñas? Revista Latinoamericana de Ciencias Sociales, Niñez y Juventud, 16(1), 227-237. https://doi.org/10.1160o/1692715x.16113

Moreno-Zavaleta, M. T., \& Granada-Echeverri, P. (2014). Interacciones vinculares en el sistema de cuidado infantil. Revista Latinoamericana de Ciencias Sociales, Niñez y Juventud, 12(1), 121-139. https://doi.org/10.1160o/1692715x.1216052513

Muñoz, J. M., Braza, P., Carreras, R., Braza, F., Azurmendi, A., Pascual-Sagastizábal, E. ... Sánchez-Martín, J. (2017). Daycare center attendance buffers the effects of maternal authoritarian parenting style on physical aggression in children. Frontiers in Psychology 8, 391, 1-7. https://doi.org/10.3389/fpsyg.2017.00391 
Muñoz, M., \& Santelices, M. P. (en prensa). Programa PAS: efectos sobre la respuesta sensible del personal de salas cuna. Revista Latinoamericana de Psicología.

NICHD Early Child Care Network. (2002). Early child care and children's development prior to school entry: results from the NICHD Study of Early Child Care. American Educational Research Journal, 39, 133-164. https://doi.org/10.3102/00028312039001133 Nóblega, M., Conde, G., Núñez del Prado, J., Bárrig, P., Marinelli, F., Alcántara, N., \& Cárcamo, R. A. (2019). Evidencias de validez de constructo y de criterio de la escala Massie-Campbell de apego durante estrés (ADS). Acta Colombiana de Psicología, 22(1), 129-140. https://doi.org/10.14718/acp.2019.22.1.7

Noboa-Hidalgo, G. E., \& Urzúa, S. S. (2012). The effects of participation in public child care centers: Evidence from Chile. Journal of Human Capital, 6, 1-34. https://doi.org/ $10.1086 / 664790$

Olhaberry, M. (2011). Calidad de la interacción madre-hijo(a), asistencia a salas cuna y la importancia de la edad de ingreso: estudio comparativo en familias monoparentales chilenas. Revista Argentina de Clínica Psicológica, 20, 161-173.

Pallini, S., Baiocco, R., Baumgartner, E., Bellucci, M. T., \& Laghi, F. (2017). Attachment in childcare centers: Is it related to toddlers' emotion regulation and attentive behavior? Child Indicators Research, 10, 205-220. https://doi.org/10.1007/s12187-016-9371-5

Pluess, M., \& Belsky, J. (2009). Differential susceptibility to rearing experience: The case of childcare. Journal of Child Psychology and Psychiatry, 50, 396-404. https://doi.org/ 10.1111/j.1469-7610.2008.01992.x

Salinas-Quiroz, F., \& Posada, G. (2015). MBQS: Método de evaluación para intervenciones en apego dirigidas a primera infancia. Revista Latinoamericana de Ciencias Sociales, Niñez y Juventud, 13(2), 1051-1063. https://doi.org/10.1160o/1692715x.13235280514

Santelices, M. P., Carvacho, C., Farkas, C., León, F., Galeguillos, F., \& Himmel, E. (2012). Medición de la sensibilidad del adulto con niños de 6 a 36 meses de edad: construcción y análisis preliminares de la escala de sensibilidad del adulto, ESA. Terapia Psicológica, 30, 19-29. https://doi.org/10.4067/s0718-48082012000300003

Santelices, M., \& Olhaberry, M. (2009). Asistencia temprana a salas cuna y patrones de apego infantil: una revisión. Summa Psicológica UST, 6, 101-111. https://doi.org/ 10.18774/448x.2009.6.54

van der Horst, F. C. P., \& van der Veer, R. (2009). Changing attitudes towards the care of children in hospital: A new assessment of the influence of the work of Bowlby and Robertson in Britain, 1940-1970. Attachment \& Human Development, 11, 119-142. https://doi.org/10.1080/14616730802503655 